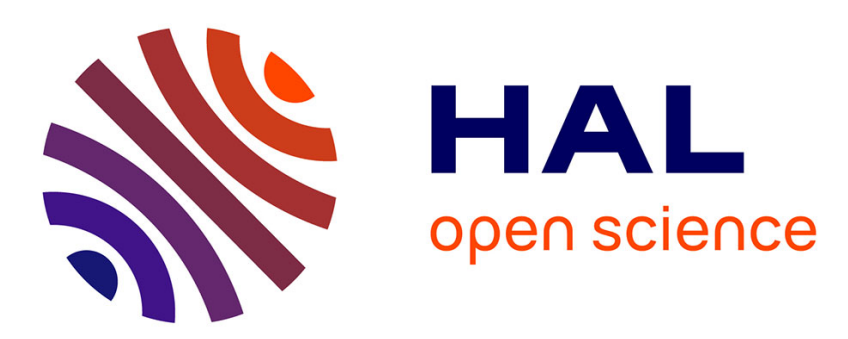

\title{
Motion of a vibrating string in the presence of a convex obstacle: A free boundary problem \\ Henri Cabannes
}

\section{To cite this version:}

Henri Cabannes. Motion of a vibrating string in the presence of a convex obstacle: A free boundary problem. Mathematical Methods in the Applied Sciences, 1987, 9 (1), pp.276-297. 10.1002/mma.1670090122 . hal-01527092

\section{HAL Id: hal-01527092 \\ https://hal.science/hal-01527092}

Submitted on 23 May 2017

HAL is a multi-disciplinary open access archive for the deposit and dissemination of scientific research documents, whether they are published or not. The documents may come from teaching and research institutions in France or abroad, or from public or private research centers.
L'archive ouverte pluridisciplinaire HAL, est destinée au dépôt et à la diffusion de documents scientifiques de niveau recherche, publiés ou non, émanant des établissements d'enseignement et de recherche français ou étrangers, des laboratoires publics ou privés. 


\title{
Motion of a Vibrating String in the Presence of a Convex Obstacle: A Free Boundary Problem
}

\author{
H. Cabannes, Paris
}

\begin{abstract}
Here we study the motion of a vibrating string in the presence of an arbitrary obstacle. We show that if the string always rebounds on the concave parts of the obstacle, it can either rebound or roll on the convex parts. The latter is the case if the velocity of the siring is null at the contact point just before contact, or if the contact point propagates at a characteristic speed. Four examples are given. The three first correspond to the same obstacle, a sinusoidal arc, but with different initial conditions. In the first case, the string rebounds on the whole of the abstacle and the motion is explicitly determined when it is periodic. In the second case, the string rolls on the convex part of the obstacle up to the inflexion point and then rebounds on the concave part and unwinds on the convex part. In the third case, the string is initially at rest on the obstacie; then it instantaneously leaves the concave part while it unwinds progressively on the convex part. The fourth case is similar to the third but with a different obstacle; the motion, which is periodic, is determined explicitly.
\end{abstract}

\section{Introduction}

Problems related to the study of the motion of a vibrating string in presence of an obstacle were first considered by Amerio and Prouse in 1975 [3]. Since then about fifty papers devoted to the subject have been published. The cases most often considered concerne straight-line or point-mass obstacles [5], [10]. In the case of curvilinear obstacles a fundamental difference appears between concave obstacles (for an observer on the string) and convex obstacles. If the obstacle is concave, the string rebounds instantaneously, while if the obstacle is convex the string can either rebound or can roll up while remaining in contact with the obstacle for some time. This last behaviour also appears when the string vibrates in the presence of a point-mass obstacle [11], [5]. There are only a few papers related to the cases of curvilinear obstacles, in particular one by Schatzman [12] on concave obstacles, while Burridge et al. [4], and Amerio [2] considered convex obstacles. The reference [4] treats an explicit solution of a vibrating string against a parabolic obstacle with an inelastic reflection law; this solution represents the behaviour of the strings of an Indian musical instrument. In reference [2] Amerio studied in great detail and generality persistent contacts of the string with obstacle, contacts which exist only for convex obstacles. 
The purpose of the present paper is to contribute to the study of the motion of a vibrating string in presence of convex obstacles. In the first part, $\$ \S 2,3,4$, we study the conditions of rebound and the conditions of wrapping of the string on the obstacle. A priori one can think that the string wraps around when it comes in contact with the obstacle with a null velocity. We will show that even if its velocity is not zero, there are cases in which the string wraps around rather that rebounds because rebound is not possible. If the obstacle is convex in one region and concave in another, these regions being separated by an inflexion point, then when the end of the part of the string wrapped on the obstacle crosses the inflexion point and reaches the concave part, there is a rebound on the concave part while the string unwraps on the convex part. In the second part, $\S 5,6,7$ and 8 , examples are given. The obstacle is either a sinusoidal arc corresponding to one period $(\$ \$ 5,6$ and 7), or a portion of a sinusoid corresponding to one and a half period $(\S 8)$. The first obstacle possesses a centre of symmetry, which is an inflexion point; half of the obstacle is convex, the other half is concave. The second obstacle possesses an axis of symmetry with two inflexion points. In all cases the reflection of the string on the obstacle takes place with conservation of energy. For both obstacles, periodic motions have been obtained, the string being initially at rest with an appropriate shape ( $\$ 5$ and 8); if the initial conditions are different, the periodic character of the motion disappears. In $\S 6$, we have obtained a solution for which the string wraps on the convex part of the sinusoid, and then unwraps when the extremity of the arc in contact reaches the inflexion point. Finally, in $\$ \$ 7$ and 8 , the string is at rest at a given time on the obstacles; it then leaves the concave parts instantaneously and the convex parts progressively. The determination of the limits of the arcs of the string in contact with the obstacle during the wrapping and during the unwrapping is a free boundary problem, as Amerio has already shown [2].

\section{Statement of the problem}

The motion of a string is defined by the position, at time $t$, of a point $M$ with curvilinear abscissa $s$. The string being assumed inextensible, $s$ is the curvilinear abscissa for the initial state, and for any arbitrary state; we then have $M=M(s, t)$ and $|\partial \vec{M} / \partial s|=1$. We assume that there is no torque, but that external forces of density $\vec{f}$ are applied on the string. If the string is also assumed perfectly flexible, the constraint at each point is a vector $\vec{T}$ tangent to the string; $\vec{T}=T(\partial \vec{M} / \partial s)$. The equations of motion are

$$
\varrho \frac{\partial^{2} \vec{M}}{\partial t^{2}}=\frac{\partial}{\partial s}\left(T \frac{\partial \vec{M}}{\partial s}\right)+\vec{f}, \quad\left|\frac{\partial \vec{M}}{\partial s}\right|=1,
$$

where $\varrho(s)$ is the density of the string. 
These equations constitute a system of four equations, with four unknowns. The tension is assumed positive, which corresponds to a strained string.

During the motion the string can strike against a fixed obstacle. The only force applied on the string is, by assumption, the reaction of the obstacle, so that in the absence of contact, $f=0$, whereas during a persistent contact

$$
\vec{f}=-\frac{\partial}{\partial s}\left(T \frac{\partial \vec{M}}{\partial s}\right)=-T \frac{\partial^{2} \vec{M}}{\partial s^{2}}-\frac{\partial T}{\partial s} \frac{\partial \vec{M}}{\partial s} .
$$

It follows from relation ( $\left.1^{\prime}\right)$ that the reaction is in the osculator plane, and in the case of two dimensional motion it is in the plane of the motion. From now on, we will only consider this last case. With respect to the tangent to the string the reaction is on the side opposed to $\left(\partial^{2} \vec{M} / \partial s^{2}\right)$, that is on the side opposite to the concavity (figures 1). To satisfy the laws of action of contact, the reaction must be directed from the obstacle to the string; this will be the case, by relation (1'), only if the string is on the side opposed to $\left(\partial^{2} \vec{M} / \partial s^{2}\right)$, that is if the obstacle is convex for an observer on the string because we consider only strained strings, what corresponds to positive values of $T$. This means that with a concave obstacle a persistent contact is not possible and that the string rebounds instantaneously on the obstacle.

This possibility or impossibility of a persistent contact is at the origin of the fundamental difference between oscillations in the presence of a convex obstacle and oscillations in the presence of a concave obstacle. At all regular points of the obstacle the second derivative $\left(\partial^{2} \vec{M} / \partial s^{2}\right)$ and therefore the force density $\vec{f}$ have a finite modulus. At an angular point the obstacle exerts on the string an action which is a concentrated force,

$$
\vec{F}=\lim _{\varepsilon \rightarrow 0} \int_{s_{0}-\varepsilon}^{s 0+\varepsilon} \frac{\partial}{\partial s}\left(T \frac{\partial \vec{M}}{\partial s}\right) \mathrm{d} s=-\left[T \frac{\partial \vec{M}}{\partial s}\right],
$$

The notation $[Q(s)]=Q(s+0)-Q(s-0)$ denotes the discontinuity of the function $Q(s)$ at the point abscissa $s$.

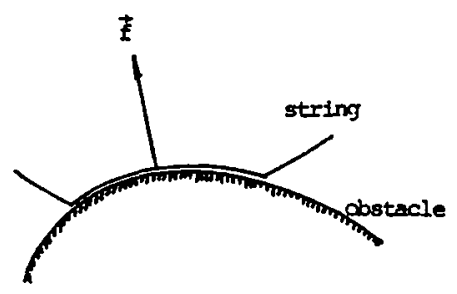

Figure 1.a Convex obstacle

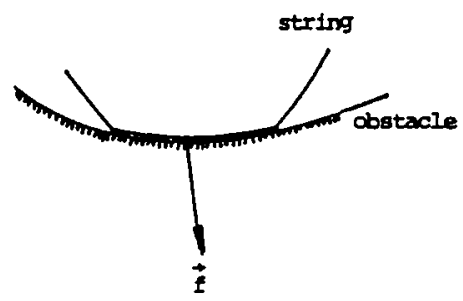

Figure 1.b Concave obstacle 
We consider in this paper small motions of a vibrating string. The string, assumed homogeneous, oscillates in a plane related to an orthonormal reference system Oxu. In its equilibrium position the string is on the segment $A B$ of the $x$-axis (all the variables are non-dimensional). The points $A$ and $B$, of abscissa $x= \pm 0.5$, are fixed points of the string. The small motions of the string near the equilibrium position are governed by the classical equation of vibrating strings, obtained by the linearisation of equation (1). Denoting the ordinate of the point with abscissa $x$, at time $t$, by $u(x, t)$, we have, in the absence of contact,

$$
\begin{aligned}
& \square u \equiv \frac{\partial^{2} u}{\partial t^{2}}-\frac{\partial^{2} u}{\partial x^{2}}=0 \\
& u\left( \pm \frac{1}{2}, t\right)=0 \quad \text { for } t \geqslant 0 .
\end{aligned}
$$

The string is subjected to oscillations above a curvilinear obstacle $u=\Phi(x)$ which it can hit during the motion. In the next $\S \S$ we will introduce the characteristic coordinates $\xi=t+x$ and $\eta=t-x$.

In the linear theory the tension is constant, and from equation (1') the density of the reacting forces during a persistent contact is equal to the opposite of the second derivative of the function $\Phi(x): f=-\Phi^{\prime \prime}(x)$. At an angular point of the obstacle the reaction is a concentrated force

$$
F=-\left[\Phi^{\prime}(x)\right] \text {. }
$$

\section{Condition for the string to rebound on the obstacle}

For an arbitrary obstacle (concave or convex), we assume that the string in motion $u=u(x, t)$ encounters the obstacle at a point $Q$ of abscissa $x=\sigma(t)$. We denote by $\tau(x)$ the inverse function of the function $\sigma: \sigma \circ \tau=$ identity. We have of course

$$
u(x, \tau(x)) \equiv \Phi(x) .
$$

At the point $Q$ the string rebounds on the obstacle according to a certain law of reflection. The velocity of the string at point $Q$, negative before the contact, becomes positive:

$$
\frac{\partial u}{\partial t}(x, \tau+0)=-\lambda(x) \frac{\partial u}{\partial t}(x, \tau-0) .
$$

The reflection coefficient $\lambda(x)$ is given, positive or null. In the $x, t$ plane (figure 2), the curve $x=\sigma(t)$, called the contact curve, is a part of the line of influence (cf. [1], [4]) on which $|\mathrm{d} \tau / \mathrm{d} x| \leqslant 1$, that is $|\dot{\sigma}(t)| \geqslant 1$; in other words the point $Q$ moves at a supersonic speed, or exceptionally at sonic speed. The contact curve is part of the boundary of the domain $u(x, t) \leqslant \Phi(x)$ and the line of influence is formed by arcs of contact and their characteristic tangents. 


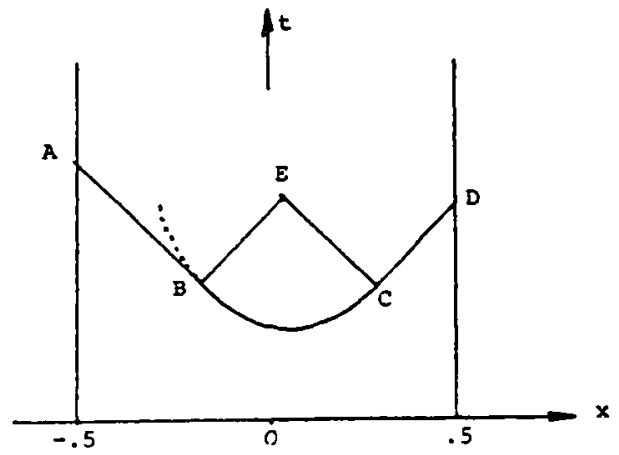

Figure 2 Line of influence

In figure 2, the line of influence $A B C D$ is composed of a characteristic segment $A B$ which is not an arc of contact, an arc of contact $B C$ on which $|\dot{\sigma}(t)|>1$, and an arc of contact $C D$ which is characteristic and on which $\dot{\sigma}(t)=1$. The existence of characteristic arcs of contact is an exception and corresponds to particular initial conditions. The boundary of the domain $u(x, t) \leqslant \Phi(x)$ is continued by a dotted line on which $|\dot{\sigma}(t)|<1$.

On the arc $B C$ of the contact curve one knows the values of $u(x, t)$ and $(\partial u / \partial t)(x, t)$; therefore the function $u(x, t)$ is determined (Cauchy problem) in the domain $B C E$ bounded by the arc $B C$, the characteristic $B E(t-x$ $\left.=t_{B}-x_{B}\right)$, and the characteristic $C E\left(t+x=t_{C}+x_{C}\right)$.

On the characteristic segment $C D$ of the contact curve one always has $u(x, t)=\Phi(x)$, but relation (6) cannot be satisfied. In fact, the arc $C D$ being characteristic, the motion of the string past $C D$ is determined by the values of the function $u(x, t)$ on the two characteristics issuing from the point $C, C D$ and $C E$. We deduce in particular the value of the velocity $\partial u / \partial t$, and (by applying relation (6)), the value of the reflection coefficient $\lambda(x)$, which cannot be given, but depends on the motion of the string.

On the non-characteristic arcs of contact, we will adopt the function $\lambda(x)=1$ which corresponds to a perfect elastic reflection; and to include the two cases $|\dot{\sigma}(t)|>1$ and $|\dot{\sigma}(t)|=1$, we will write the law of reflection of the string in the form

$$
\left(\dot{\sigma}-\frac{1}{\dot{\sigma}}\right)\left[\left(\frac{\partial u}{\partial t}\right)^{2}\right]=0 .
$$

We deduce from this law the following result.

Theorem. During the motion, the total energy of the string (kinetic energy and potential energy) remains constant.

This theorem is expressed by the equation 


$$
E(t)=\int_{-1 / 2}^{1 / 2}\left\{\left(\frac{\partial u}{\partial t}\right)^{2}+\left(\frac{\partial u}{\partial t}\right)^{2}\right\} \mathrm{d} x=\text { const. }
$$

We assume first that the string encounters the obstacle in a finite numbers of points $Q_{1}, Q_{2}, \ldots Q_{n}$ of abscissae $\sigma_{1}(t), \sigma_{2}(t), \ldots$ with $-0.5<\sigma_{1}<\sigma_{2} \ldots$ $\sigma_{n}<0.5$.

\section{Then}

$$
\begin{aligned}
& \frac{\mathrm{d}}{\mathrm{d} t} \int_{\sigma_{1}(t)}^{\sigma_{+1}(t)}\left\{\left(\frac{\partial u}{\partial t}\right)^{2}+\left(\frac{\partial u}{\partial x}\right)^{2}\right\} \mathrm{d} x=\dot{\sigma}_{i+1}(t)\left\{\left(\frac{\partial u}{\partial t}\right)^{2}+\left(\frac{\partial u}{\partial x}\right)^{2}\right\}\left(\sigma_{i+1}-0, t\right) \\
& -\dot{\sigma}_{i}(t)\left\{\left(\frac{\partial u}{\partial t}\right)^{2}+\left(\frac{\partial u}{\partial x}\right)^{2}\right\}\left(\sigma_{i}+0, t\right)+\int_{\sigma_{1}}^{\sigma_{i+1}} \frac{\partial}{\partial t}\left\{\left(\frac{\partial u}{\partial t}\right)^{2}+\left(\frac{\partial u}{\partial x}\right)^{2}\right\} \mathrm{d} x,
\end{aligned}
$$

and, by virtue of equation (3), one has

$$
\frac{\partial}{\partial t}\left\{\left(\frac{\partial u}{\partial t}\right)^{2}+\left(\frac{\partial u}{\partial x}\right)^{2}\right\}=2 \frac{\partial}{\partial x}\left\{\frac{\partial u}{\partial t} \cdot \frac{\partial u}{\partial x}\right\},
$$

so that the last line of the right hand side of equation (9) can also be written

$$
2\left(\frac{\partial u}{\partial t} \cdot \frac{\partial u}{\partial x}\right)\left(\sigma_{i+1}-0, t\right)-2\left(\frac{\partial u}{\partial t} \cdot \frac{\partial u}{\partial x}\right)\left(\sigma_{i}+0, t\right) .
$$

As $(\partial u / \partial t)$ vanishes for $x= \pm 0.5$, we obtain by addition of all of equations (9), with $\sigma_{0}(t)=-0.5$ and $\sigma_{n+1}(t)=0.5$,

$$
\frac{\mathrm{d} E}{\mathrm{~d} t}=-\sum_{i=1}^{n}\left\{\dot{\sigma}_{i}\left[\left(\frac{\partial u}{\partial t}\right)^{2}+\left(\frac{\partial u}{\partial x}\right)^{2}\right]+2\left[\frac{\partial u}{\partial t} \cdot \frac{\partial u}{\partial x}\right]\right\}
$$

where the discontinuities are assumed at the points $x=\sigma_{i}(t)$. The function being continuous, in particular for $x=\sigma_{i}(t)$, we have $u\left(\sigma_{i}+0, t\right) \equiv u(\sigma i-$ $0, t)$ and therefore

$$
\begin{aligned}
& \left(\dot{\sigma}_{i} \frac{\partial u}{\partial x}+\frac{\partial u}{\partial t}\right)^{2}\left(\sigma_{i}+0, t\right)=\left(\dot{\sigma}_{i} \frac{\partial u}{\partial x}+\frac{\partial u}{\partial t}\right)^{2}\left(\sigma_{i}-0, t\right) \\
& \dot{\sigma}_{i}\left[\left(\frac{\partial u}{\partial x}\right)^{2}\right]+2\left[\frac{\partial u}{\partial x} \cdot \frac{\partial u}{\partial t}\right]+\frac{1}{\dot{\sigma}_{i}}\left[\left(\frac{\partial u}{\partial t}\right)^{2}\right]=0 \\
& \frac{\mathrm{d} E}{\mathrm{~d} t}=-\sum_{i=1}^{n}\left(\dot{\sigma}_{i}-\frac{1}{\dot{\sigma}_{i}}\right)\left[\left(\frac{\partial u}{\partial t}\right)^{2}\right]\left(\sigma_{i}, t\right)
\end{aligned}
$$

and by virtue of relation (7) $(\mathrm{d} E / \mathrm{d} t)$ is zero. When the string is in contact with the obstacle not only on isolated points, but also on some arcs, the result remains valid. We have in fact

$$
\frac{\mathrm{d}}{\mathrm{d} t} \int_{\sigma_{1}}^{\sigma_{1+1}} \Phi^{\prime 2}(x) \mathrm{d} x=\dot{\sigma}_{i+1} \Phi^{\prime 2}\left(\sigma_{i+1}-0\right)-\dot{\sigma}_{i} \Phi^{\prime 2}\left(\sigma_{i}+0\right) .
$$


This formula is exactly formula (9) if we note that on a contact arc, $\sigma_{i} \leqslant x \leqslant \sigma_{i+1}$, we have $(\partial u / \partial t)=0$ and $(\partial u / \partial x)=\Phi^{\prime}(x)$.

\section{Wrapping and unwrapping of the string on the obstacle}

When the string hits a concave obstacle, it always rebounds; when it hits a convex obstacle, it can either rebound or wrap up and remain in contact with the obstacle for some time. We assume that the motion of the string above the obstacle $u=\Phi(x)$ is the free oscillation $u(x, t)=w(x, t)$. If $\Phi( \pm 0.5)$ is strickly negative, the domain $(\mathscr{D}), w(x, t) \leqslant \Phi(x)$, is entirely in the region $t \geqslant 0,|x|<0.5$ and the free oscillation ends on the line of influence (line $A^{\prime} A B C D D^{\prime}$ in figure 3-a) composed of arcs of the boundary $t=\tau(x)$ of the domain $(\mathscr{D})(\operatorname{arcs} A B$ and $C D$ in figure 3-a) and of characteristic tangents $\left(A^{\prime} A, B C\right.$ and $\left.D D^{\prime}\right)$.

On the arcs $A B$ and $C D$, on which there is a contact between the string and the obstacle, one has $\left|\tau^{\prime}(x)\right|<1$; the contact point of the string with the obstacle propagates on the string with supersonic speed; exceptionally the boundary of the domain $(\mathscr{D})$ can be composed of characteristic segments.

We assume now that one of the extremities of the string is on the obstacle, $\Phi(-0.5)=0$. The boundary of the domain $(\mathscr{D})$ can also meet the axis $x=-0.5$, and we assume that this is the case at the point $A(x=-0.5$, $\left.t=t_{A}\right)$ : figure 3-b. If, near the point $A$, on the boundary $t=\tau(x)$ of the domain $(\mathscr{D}), \tau^{\prime}(x)$ is smaller than 1 , or equal to 1 , the former conclusions remain valid: the free oscillation ends on the line of influence. In the opposite case (figure 3-b) new phenomena appear.

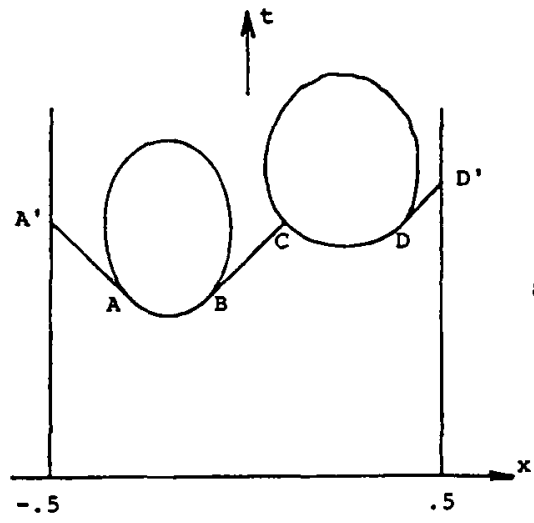

Figure 3.a

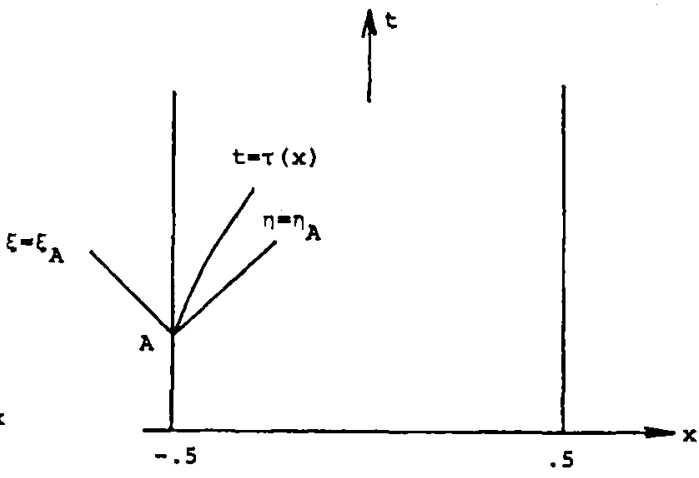

Figure 3.b

To study the curve $t=\tau(x)$ near the point $A$, we note: $x=-0.5+$ $\delta x, t=t_{A}+\delta t$ ( $\delta x$ and $\delta t$ positive) and we expand in series the two sides 
of the equality $w(x, t)=\Phi(x)$. The functions $w(x, t)$ and $\Phi(x)$ being assumed twice continuously differentiable, we obtain

$$
\begin{aligned}
& w\left(-0.5, t_{A}\right)+\frac{\partial w}{\partial x} \delta x+\frac{\partial w}{\partial t} \delta t \\
& \quad+\frac{1}{2}\left\{\frac{\partial^{2} w}{\partial x^{2}}(\delta x)^{2}+2 \frac{\partial^{2} w}{\partial x \partial t} \delta x \cdot \delta t+\frac{\partial^{2} w}{\partial t^{2}}(\delta t)^{2}\right\}+\ldots \\
& =\Phi(-0.5)+\Phi^{\prime}(-0.5) \delta x+\frac{1}{2} \Phi^{\prime \prime}(-0.5)(\delta x)^{2}+\ldots
\end{aligned}
$$

The values of functions are the values at the point $A\left(x=-0.5, t=t_{A}\right)$. The boundary condition satisfied by the function $w(x, t)$ for $x=-0.5$ gives

$$
\begin{aligned}
& w(-0.5, t)=0, \quad \frac{\partial w}{\partial t}(-0.5, t)=0 \\
& \frac{\partial^{2} w}{\partial t^{2}}(-0.5, t)=\frac{\partial^{2} w}{\partial x^{2}}(-0.5, t)=0 .
\end{aligned}
$$

We deduce from relations (14) and (15) that

$$
\frac{\partial w}{\partial x}\left(-0.5, t_{A}\right)=\Phi^{\prime}(-0.5) \quad 2 \frac{\partial^{2} w}{\partial x \partial t}\left(-0.5, t_{A}\right) \frac{d \tau}{d x}=\Phi^{\mu}(-0.5) .
$$

The first relation (16) determines the time $t_{A}$, the second the slope at this point of the curve $t=\tau(x)$. Otherwise, as the string oscillates above the obstacle, the velocity of the string just before contact is negative. Taking in account the relations (15) one has

$$
\frac{\partial w}{\partial t}\left(-0.5+\delta x, t_{A}+\delta t\right)=\frac{\partial^{2} w}{\partial x \partial t}\left(-0.5, t_{A}\right) \delta x+\ldots<0
$$

As a result, the second derivate $\Phi^{\prime \prime}(-0.5)$ has a sign opposite to the sign of $\tau^{\prime}(-0.5)$. In particular the case $\tau^{\prime}(-0.5)>1$ can appear only if $\Phi^{\prime \prime}(-0.5)$ is negative, that is to say, if the obstacle is convex near the point $x=-0.5$, $u=\Phi(-0.5)=0$. In that case rebound is not possible; in fact if the string rebounds, the motion following contact is determined by the rebound conditions. The determination of the motion is a Cauchy problem, the solution of which is defined in a domain of the $x, t$ plane bounded by the contact curve $t=\tau(x)$ and by two characteristics. One of these is the characteristic $t+x=t_{A}+x_{A}$, and the domain contains a portion of the axis $x=-0.5$; except in isolated cases, the condition $u(-0.5, t)=0$ is not satisfied. We deduce from this impossibility that the free oscillation ends, not on the boundary of the domain (D) , but on the characteristic issuing from the point $A: t-x=t_{A}-x_{A}$. On the other side of this characteristic, the function $u(x, t)$ $=\tilde{u}(\xi, \eta)$ which represents the motion of the string has the form 


$$
\tilde{u}(\xi, \eta)=\tilde{w}\left(\xi, \eta_{A}\right)+g(\eta) \text { with } g\left(\eta_{A}\right)=0 ;
$$

the function $g(\eta)$ is determined by the conditions which express the wrapping of the string on the obstacle.

Those conditions are: on an unknown curve, the equation of which we write $t=\tau(x)$, one must have $u(x, \tau(x)) \equiv \Phi(x)$ and $(\partial u / \partial t)(x, \tau(x)) \equiv 0$ :

$$
\tilde{w}\left(\tau+x, \eta_{A}\right)+g(\tau-x) \equiv \Phi(x), \quad \frac{\partial \tilde{w}}{\partial \xi}\left(\tau+x, \eta_{A}\right)+g^{\prime}(\tau-x) \equiv 0 .
$$

This is a free boundary problem, as showed by Amerio [10]; the solution is simple. The system (18) is a system of two equations with two unknowns: the functions $\tau(x)$ and $g(\eta)$. Taking the derivative of the first equation, and eliminating $g^{\prime}(\tau-x)$, we obtain the equation

$$
2 \frac{\partial \tilde{w}}{\partial \xi}\left(\tau+x, \eta_{A}\right)=\Phi^{\prime}(x)
$$

which determines the function $\tau(x)$, the free boundary, that is to say the trajectory of the extremity of the arc of the string rolled round the obstacle. When the function $\tau(x)$ is known, the first equation (18) gives the value of the function $g(\eta)$.

The unwrapping of the string appears in similar conditions, which will be examined in the examples of $\S 6,7$ and 8 .

\section{$5 \quad$ First example}

We consider an obstacle formed by a sinusoidal arc:

$$
u=\Phi(x)=-\tan \frac{\theta}{2} \sin \pi x \cdot \cos \pi x, \quad-\frac{1}{2} \leqslant x \leqslant \frac{1}{2}
$$

where $\theta$ is a constant between 0 and $\pi / \pi$. The obstacle is convex for $x<0$, concave for $x>0$ and has an inflexion point at $x=0$. At the initial time we assume that the string is at rest in the position $u(x, 0)=\cos \pi x$. In the absence of the obstacle the motion is a free oscillation

$$
u(x, t)=w(x, t)=\cos \pi x \cdot \cos \pi t .
$$

When $\theta=0$ (straight obstacle on the equilibrium position of the string), the motion of the string is $u(x, t)=|w(x, t)|=\cos \pi x \cdot|\cos \pi t|$. We first assume $0<\theta<\pi / 2$. At the beginning, the motion of the string is free oscillation $u(x, t)=w(x, t)$ up to the first curve of contact

$$
\cos \pi t+\tan \frac{\theta}{2} \cdot \sin \pi x=0
$$


This curve (figure 4) joins the points $A(x=-0.5, \cos \pi t=\tan (\theta / 2))$ and $B(x=-0.5, \cos \pi t=-\tan (\theta / 2))$; on the $\operatorname{arc} A B$

$$
\frac{\mathrm{d} t}{\mathrm{~d} x}=\tan \frac{\theta}{2} \quad \frac{\cos \pi x}{\sin \pi t} \quad 0 \leqslant \frac{\mathrm{d} t}{\mathrm{~d} x} \leqslant \tan \frac{\theta}{2}<1
$$

because

$$
\begin{aligned}
& 0 \leqslant \sin ^{2} \pi x \cdot\left(1-\tan ^{2} \frac{\theta}{2}\right) \\
& \cos ^{2} \pi x \leqslant 1-\sin ^{2} \pi x \cdot \tan ^{2} \frac{\theta}{2}=\sin ^{2} \pi t .
\end{aligned}
$$

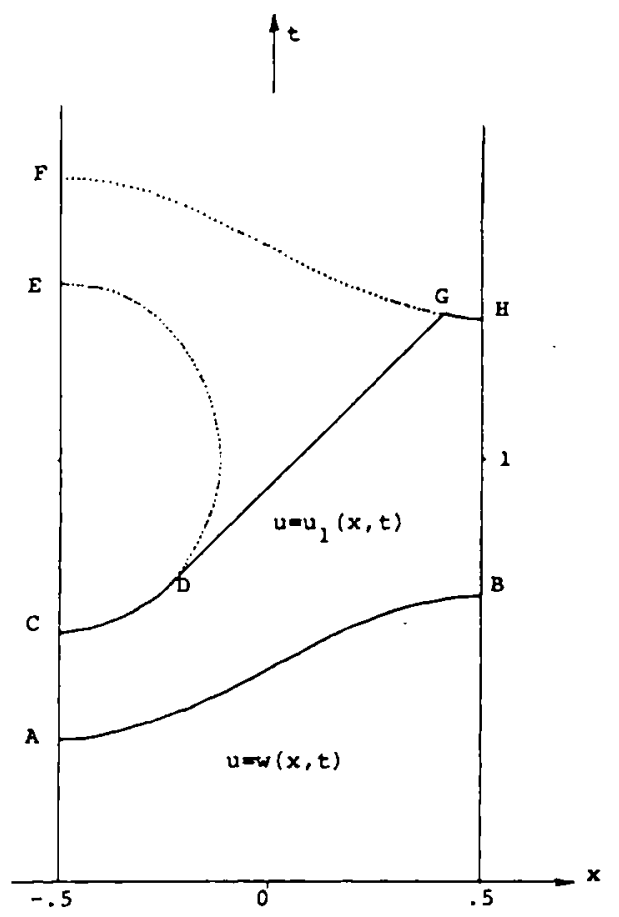

Figure 4 Obstacle $u=\Phi(x)=-\tan \frac{\theta}{2} \cdot \sin \pi x \cdot \cos \pi x$

The first line of influence is then formed by the arc $A B$, which containts the point $x=0, t=0.5$ for all values of the parameter $\theta$. To determine the motion of the string $u(x, t)=u_{1}(x, t)$ after the first contact we let

$$
u_{1}(x, t)=\tilde{u}_{1}(\xi, \eta)=f(\xi)+g(\eta)
$$


where $\xi=t+x$ and $\eta=t-x$ denote the characteristic variables. On the arc $A B$, we have

$$
\sin \pi \xi=\frac{\sin \pi \eta-\sin \theta}{1-\sin \theta \sin \pi \eta}, \quad \frac{\mathrm{d} \xi}{\mathrm{d} \eta}=-\frac{1+\sin \theta \cdot \sin \pi \eta}{\cos \theta}
$$

Letting $u_{1} \equiv w,\left(\partial u_{1} / \partial t\right) \equiv-(\partial w / \partial t)$ on the arc $A B$ the functions $f(\xi)$ and $g(\eta)$ are obtained:

$$
f(\xi)+g(\eta)=\frac{1}{2}\{\cos \pi \xi+\cos \pi \eta\}
$$

$$
f^{\prime}(\xi)+g^{\prime}(\eta)=\frac{\pi}{2}\{\sin \pi \xi+\sin \pi \eta\} .
$$

Taking the derivative of the arc $A B$ of the first relation in (26), we obtain, by elimination of $g^{\prime}(\eta)$,

$$
\begin{aligned}
& f^{\prime}(\xi)=\pi \cot \theta\left\{1-\frac{\cos \theta}{1+\sin \theta \cdot \sin \pi \xi}\right\}-\frac{\pi}{2} \sin \pi \xi \\
& f(\xi)=\frac{\cos \pi \xi}{2}+\operatorname{cotan} \theta\left\{\pi \xi+\theta-2 \operatorname{Arctan} \frac{\zeta+\sin \theta}{\cos \theta}\right\} \\
& \text { with } \zeta=\tan \frac{\pi \xi}{2}-1<\xi<1
\end{aligned}
$$

One then determines the function $g(\eta)$, to obtain $g(\eta)=f(-\eta)$, whence $u_{1}(x, t)=f(x+t)+f(x-t):$

$$
u_{1}(x, t)=w(x, t)+2 \operatorname{cotan} \theta\left\{\pi x-\arctan \frac{\sin \pi x+\sin \theta \cdot \cos \pi t}{\cos \theta \cdot \cos \pi x}\right\} .
$$

The function $u_{1}(x, t)$ represents the motion of the string in the region bounded by the $\operatorname{arc} A B$ and by the characteristics $\eta=\eta_{A}, \xi=\xi_{B}$. Since $u_{1}( \pm 0.5, t)$ $=0$, it represents the motion of the string after the first rebound, for -0.5 $\leqslant x \leqslant 0.5$.

The motion of the string is defined by the function $u=u_{1}$, up to the second line of influence. To define this curve we must first solve the equation

$$
u_{1}(x, t)=\Phi(x) .
$$

One solution is evidently $t=\tau(x)$, which defines the $\operatorname{arc} A B$. A second solution is $t=2-\tau(x)$ (arc $F G H$ in figure 4), since $u_{1}(x, 2-t) \equiv u_{1}(x, t)$. There is in some cases a third line $C D E$,

$$
x_{C}=-\frac{1}{2}, \quad \cos \pi t_{c}=\frac{1-2 \cos \theta}{\sin \theta} .
$$


This third line exists only when $\cos \pi t_{c}$ is less than 1 , that is if $\tan (\theta / 2)$ is greater than $1 / 3$. In this last case the line of influence is formed by the arc $C D$, the characteristic tangent $D G$, and the arc $G H$. The determination of the motion past this second line of influence cannot be made explicitly.

On the other hand, for $\tan (\theta / 2) \leqslant 1 / 3$ the function $u_{1}(x, t)$ represents the motion of the string for $\tau(x) \leqslant t \leqslant 2-\tau(x)$, and following the second rebound we have again $u(x, t)=w(x, t)$; therefore the motion of the string is periodic with period 2 .

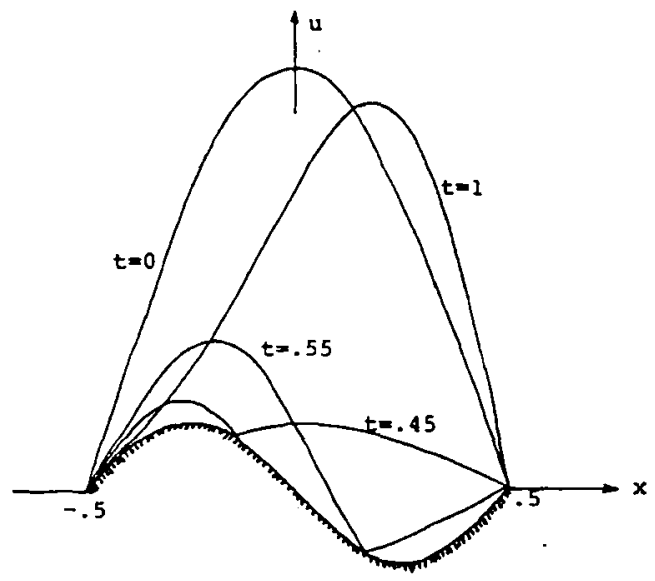

Figure 5 Obstacle $u=\Phi(x)=-\frac{1}{3} \sin \pi x \cdot \cos \pi x$

String $u(x, 0)=\cos \pi x, \frac{\partial u}{\partial t}(x, 0)=0$

Periodic motion: period $T=2$

The position of the string for various values of time is illustrated in figure 5 , which corresponds to the limit case $\tan (\theta / 2)=1 / 3$. At time $t=1$, the string is at rest in the position $u=u_{1}(x, 1)$. One can explain the results by considering that the obstacle is the sinusoidal arc $u=\Phi(x)=-\sin \pi x$. $\cos \pi x$, while the string is initially at rest in the position $u(x, 0)=\operatorname{cotan}(\theta / 2)$. $\cos \pi x$. For $\operatorname{cotan}(\theta / 2) \geqslant 3$ the motion is periodic with period 2 ; the periodic character of the motion disappears for $0<\operatorname{cotan}(\theta / 2)<3$.

In a general way, we can express the following supposition:

Conjecture. If the string, initially at rest in the position $u(x, 0)=\cos \pi x$, oscillates in the presence of the obstacle $u=\Phi(x)=\lambda \sin 2 n \pi x$ ( $n$ integer), the motion is periodic with period 2 , when $|\lambda|$ is small enough $\left.{ }^{1}\right)$.

1) The parameter $\lambda$ has no relation with the function $\lambda(x)$ introduced in Section 3 . 
In fact if $|\lambda|$ is small enough, on all the contact curve $\cos \pi x \cdot \cos \pi t$ $=\lambda \sin 2 n \pi x$, one has $|\mathrm{d} t / \mathrm{d} x|<1$ and the computation of the function $u(x, t)$ $=u_{1}(x, t)$ following the first rebound can be made by a quadrature; for $n=1$ we have obtained this function explicitly, formula (30). For an arbitrary value of $n$, one has $u_{1}(x, t)=f(x+t)+f(x-t)$ with

$$
\begin{aligned}
& f^{\prime}(\xi)=\frac{\pi}{2} \sin \pi \xi+2 n \pi \lambda \cos 2 n \pi x \\
& \xi=x+\tau(x)=x+\frac{1}{\pi} \operatorname{Arccos}(\lambda \sin 2 n \pi x / \cos \pi x) \\
& f(\xi)=-\frac{1}{2} \cos \pi \xi+\lambda \sin 2 n \pi x-\lambda^{2} \int_{0}^{\pi x} \alpha(y) \mathrm{d} y \\
& \alpha(y)=\frac{2 n \cos ^{2} 2 n y \cdot\{2 n+\tan y \cdot \tan 2 n y\}}{\cos y \sqrt{1-(\lambda \sin 2 n y / \cos y)^{2}}} .
\end{aligned}
$$

As $f(2+\xi)=f(\xi)=-f(1-\xi)$, one has $u(x, 2-t)=u(x, t)$ and, always for $|\lambda|$ small enough, there are probably no contacts between $t=t_{1}=\tau(x)$ and $t=t_{2}=2-\tau(x)$, and also the curve of the second contact is $t=2$ $-\tau(x)$. At time $t=1$, the string is at rest, and following the second rebound one again has $u(x, t)=\cos \pi x \cdot \cos \pi t$.

\section{Second example}

We consider the first example but in the limit case $\theta=\pi / 2$. The contact curve $A B$, which forms the first line of influence, is then the characteristic $\eta=0.5$. On the convex part of the obstacle $(x<0)$ the string cannot rebound and wraps on the obstacle, and thereafter we have $u(x, t)=$ $\Phi(x)$, whereas on the concave part of the string rebounds, but as the contact curve is a characteristic the coefficient of reflection is not equal to 1 , but is a function $\lambda(x)$, which will be determined. Following the first rebound we have $u(x, t)=u_{2}(x, t)$, with

$$
u_{2}(x, t)=\tilde{u}_{2}(\xi, \eta)=\frac{1}{2} \cos \pi \xi+g(\eta) \text { and } g\left(\frac{1}{2}\right)=0 .
$$

One could try to determine the function $g(\eta)$ by knowing that on the characteristic $\xi=0.5$ we have $u_{2}(x, t) \equiv \Phi(x)$, that is

$$
g(\eta)=\left(\frac{1}{2}-\frac{\eta}{2}\right)=-\frac{1}{2} \cos \pi \eta
$$


(34)

$$
\begin{array}{ll}
u_{2}(x, t)=-\sin \pi t \cdot \sin \pi x, & \text { for } \xi=\frac{1}{2} \\
\frac{\partial u_{2}}{\partial t}(x, t)=-\pi \sin ^{2} \pi x, & \text { for } \xi=\frac{1}{2} .
\end{array}
$$

The velocity of the string on the obstacle (for $\xi=0.5$ ) would be negative, which is not possible beyond the contact. Therefore the unwrapping of the string after $t=0.5$ (figure 6) cannot occur with a characteristic speed. It then happens that on a point of abscissa $x=\sigma(t)$, and on the curve $x=$ $\sigma(t)$ or $t=\tau(x)$, we must have $u_{2}(x, \tau(x)) \equiv \Phi(x)$ and, from relation (7), $\left(\partial u_{2} / \partial t\right)(x, \tau(x)) \equiv 0$. This is a free boundary problem.

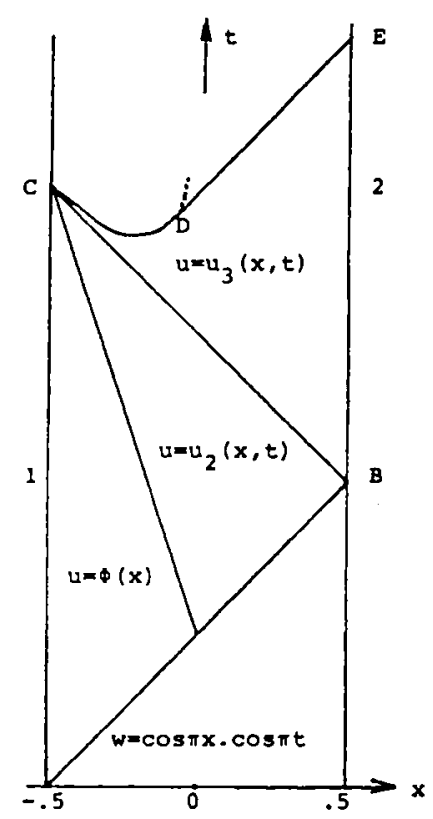

Figure 6 Obstacle $u=\Phi(x)=\sin \pi x \cdot \cos \pi x$

$$
\text { String } u(x, 0)=\cos \pi x \cdot \frac{\partial u}{\mathrm{~d} l}(x, 0)=0
$$

The functions $\tau(x)$ and $g(\eta)$ must consequently satisfy the two conditions

$$
\frac{1}{2} \cos \pi(\tau+x)+g(\tau-x)=-\sin \pi x \cdot \cos \pi x
$$

$$
-\frac{\pi}{2} \sin \pi(\tau+x)+g^{\prime}(\tau-x) \equiv 0 .
$$


By taking the derivative of the first relation and eliminating $g^{\prime}(\tau-x)$, we obtain successively

$$
\begin{aligned}
& -\frac{\pi}{2}\left(1+\frac{\mathrm{d} \tau}{\mathrm{d} x}\right) \sin \pi(\tau+x)-\left(1-\frac{\mathrm{d} \tau}{\mathrm{d} x}\right) g^{\prime}(\tau-x)=-\pi \cos 2 \pi x \\
& \sin \pi(\tau+x)=\cos \pi\left\{\frac{1}{2}-(\tau+x)\right\}=\cos 2 \pi x \\
& \tau(x)=\frac{1}{2}+2 k-x \pm 2 x, \quad k \text { integer. }
\end{aligned}
$$

As the curve $t=\tau(x)$ passes through the point $x=0, t=0.5$, the integer $k$ is zero; and as this curve is not characteristic,

$$
\begin{aligned}
& \text { (36) } \tau(x)=\frac{1}{2}-3 x \\
& \text { (37) } g(\eta)=-\sin \pi\left(\frac{1}{4}-\frac{\eta}{2}\right) \\
& \text { (38) } u_{2}(x, t)=\frac{1}{2} \cos \pi(x+t)-\sin \pi \frac{1+2 x-2 t}{4} .
\end{aligned}
$$

Therefore up to $t=0.5$ the string wraps on the convex part of the obstacle with the speed $\dot{\sigma}=1$ (figure 7). At time $t \doteq 0.5$ the extremity of the arc of the string wrapped on the obstacle reaches the inflexion point. The string

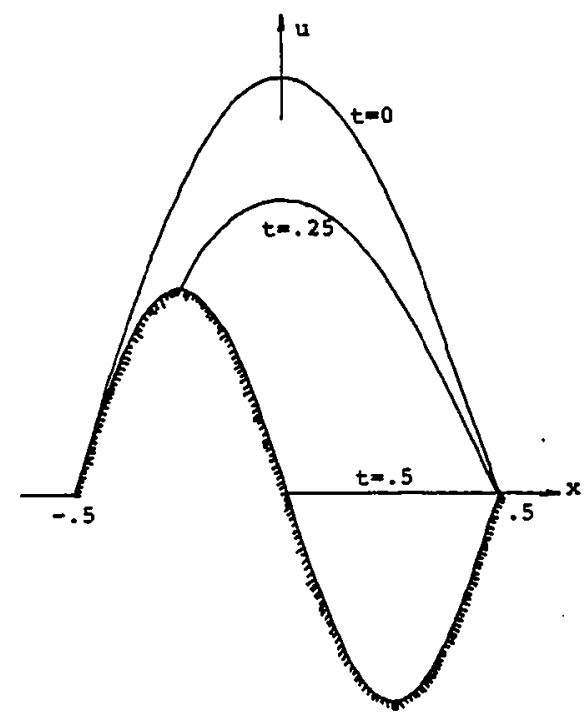

Figure 7 Wrapping of the string on the obstacle 


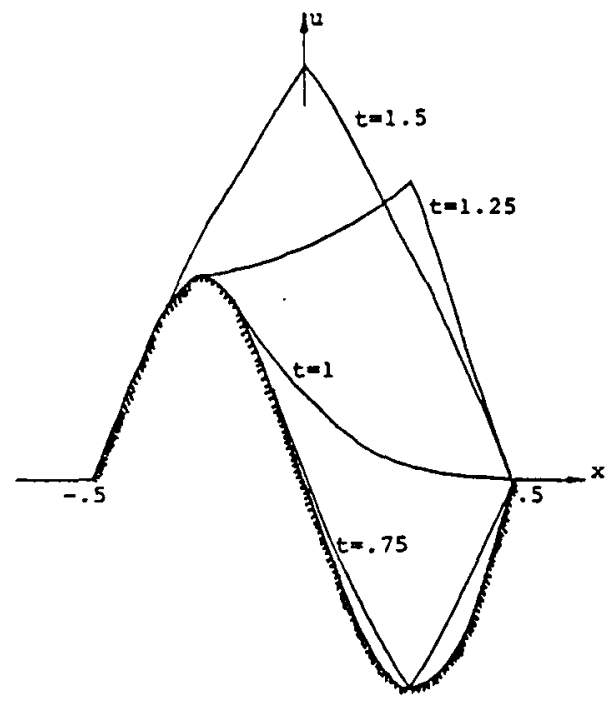

Figure 8 Rebound and unwrapping of the string on the obstacle

then rebounds on the concave part and unwraps on the convex part with the speed $\dot{\sigma}=-1 / 3$ (figure 8 ).

The motion represented by the function $u(x, t)=u_{2}(x, t)$ is valid in the triangular region bounded by the two characteristics $t-x=0.5, t+$ $x=10.5$ and the "unwrapping curve" $2 t=1-6 x$. Beyond the characteristic $t+x=10.5$, we compute

$$
u(x, t)=u_{3}(x, t)=2 \sin \frac{\pi t}{2} \cdot \sin \pi \frac{1-2 x}{4} .
$$

This new motion $u=u_{3}$ is valid until the second line of influence $C D E$ formed by the arc of contact $C D$, which corresponds to a rebound of the string on the convex part of the obstacle, and by the characteristic tangent $D E$ to the arc $C D$ (figure 6).

we have

At the first contact on the characteristic $t-x=0.5,0 \leqslant x \leqslant 5$,

$$
\frac{\partial w}{\partial t}=-\pi \cos ^{2} \pi x, \quad \frac{\partial u_{2}}{\partial t}=\pi \sin ^{2} \pi x, \quad \lambda(x)=\tan ^{2} \pi x .
$$

The value of the reflection coefficient varies from zero to infinity on the arc of contact. 
We again consider the same obstacle $u=\phi(x)=-\sin \pi x \cdot \cos \pi x$, but we assume now that the string is initially at rest on the obstacle. Immediately after the initial time the string leaves the concave part and the motion in the region $t \geqslant 0, x \leqslant 0.5, t-x \leqslant 0$ (figure 9) is defined by the solution $u(x, t)=u_{4}(x, t)$ of the equation (3), such that

(40)

$$
u_{4}(x, 0)=-\sin \pi x \cdot \cos \pi x, \quad \frac{\partial u_{4}}{\partial t}(x, 0)=0
$$

$$
u_{4}\left(\frac{1}{2}, t\right)=0 \text {; that is }
$$

$$
u_{4}(x, t)=-\frac{1}{2} \sin 2 \pi x \cdot \cos 2 \pi t=\frac{\sin 2 \pi \eta-\sin 2 \pi \xi}{4} .
$$

On the convex part of the obstacle, the string unwraps progressively, the extremity $Q$ of the arc in contact being at abscissa $x=\sigma(t)$. Determination of the function $\sigma(t)$, or the inverse function $\tau(x)$, is still a free boundary problem. The function $u(x, t)=u_{5}(x, t)$ which represents the motion of the string beyond the characteristic $t-x=0$ is determined by the conditions

$$
\begin{aligned}
& u_{5}(x, t)=-\frac{1}{4} \sin 2 \pi(t+x)+g(\eta) \\
& -\frac{1}{4} \sin 2 \pi(\tau(x)+)+g(\tau(x)-x)=-\sin \pi x \cdot \cos \pi x \\
& -\frac{\pi}{2} \cos 2 \pi(\tau(x)+x)+g^{\prime}(\tau(x)-x)=0 .
\end{aligned}
$$

Taking the derivative of the first relation (43) and eliminating $g^{\prime}(\tau+x)$ we obtain

$$
\tau(x)=-2 x \text { and } g(\eta)=\frac{3}{4} \sin \frac{2 \pi}{3} \eta .
$$

Therefore from initial time the string unwraps on the convex part of the obstacle with the speed $\dot{\sigma}=-(1 / 2)$ and we have

$$
4 u_{5}(x, t)=-\sin 2 \pi(t+x)+3 \sin 2 \pi \frac{t-x}{3} .
$$

Past the characteristic $t+x=1$, we then have $u(x, t)=u_{6}(x, t)$ with

$$
4 u_{6}(x, t)=-3 \sin 2 \pi \frac{t+x-1}{3}+3 \sin 2 \pi \frac{t-x}{3} .
$$




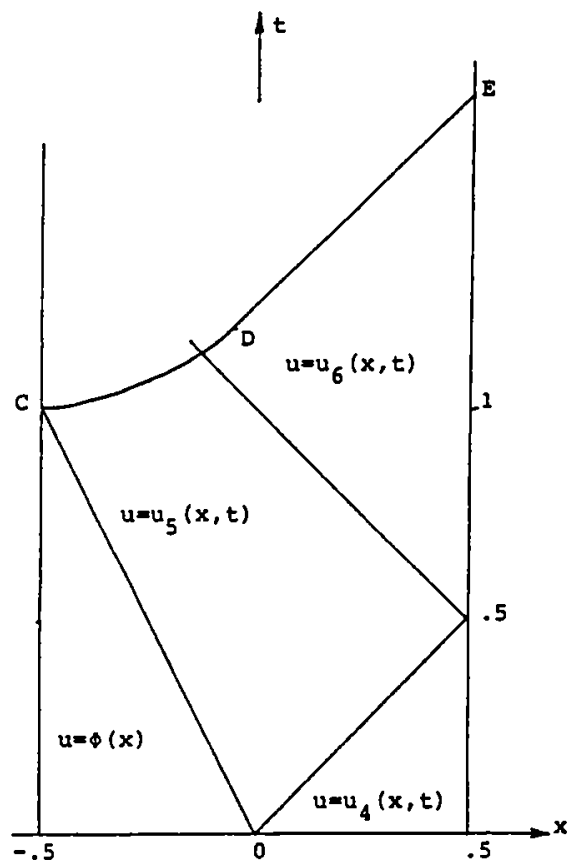

Figure 9 Obstacle $u=\Phi(x)=-\sin \pi x \cdot \cos \pi x$ String initially at rest on the obstacle

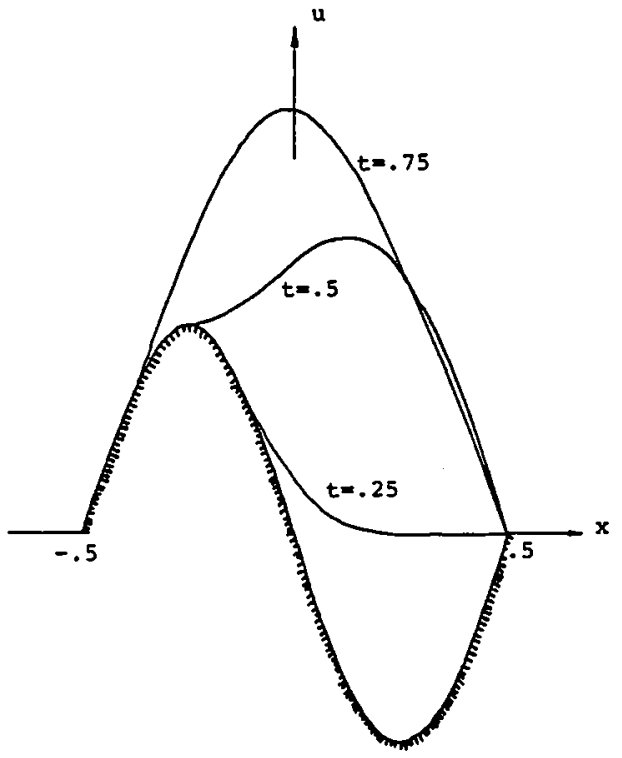

Figure 10 Obstacle $u=\Phi(x)=-\sin \pi x \cdot \cos \pi x$ String initially at rest on the obstacle 
The positions of the string at different instants are illustrated on figure 10. The regions in which the motion is represented by the functions $u_{5}(x, t)$ an $u_{6}(x, t)$ end at the first line of influence formed (figure 9) by the arc $C D$ (which corresponds to a rebound of the string on the obstacle) and by the characteristic tangent $D E$.

\section{Fourth example} axis:

We consider now a new obstacle, symmetrical with respect to the $0 u$

$$
u=\Phi(x)=-\frac{1}{3} \cos 3 \pi x=\frac{1}{3} \cos \pi x \cdot(1-2 \cos 2 \pi x) .
$$

At the initial time, the string is at rest in the position $u(x, 0)=\cos \pi x$. The free oscillation $w(x, t)=\cos \pi x \cdot \cos \pi t$ takes place in all the triangular region $0 \leqslant t \leqslant+x+0.5$; for all the region one has $w(x, t) \geqslant \Phi(x)$.

Beyond the characteristics $t \pm x=0.5$, the equation $w(x, t)=\Phi(x)$, that is to say $3 \cos \pi t=1-2 \cos 2 \pi x$, possesses a solution $t=\tau(x)$ (dotted line in figure 11) for which $|\mathrm{d} \tau / \mathrm{d} x| \geqslant 1$, if $t$ is small enough, $\pi t \leqslant$ Arc $\cos (-1 / 9)$. On the corresponding arcs, rebound is not possible, as we have shown in $\S 4$, because the solution determined by the reflection conditions does not satisfy the boundary conditions $u(0.5, t)=0$. The motion of the string beyond the characteristic $t+x=0.5$ is therefore no longer free oscillation, but is defined by a function $u(x, t)=u_{7}(x, t)$ :

$$
u_{7}(x, t)=f(\xi)+\frac{1}{2} \cos \pi \eta \text {, with } f\left(\frac{1}{2}\right)=0 .
$$

The function $f(\xi)$ is determined by the wrapping conditions of the string on the obstacle. The moving extremity (of the arc of the string in contact with the obstacle) has the (free boundary) trajectory $t=\tau(x)$ in the $x, t$ plane; and the conditions which determine the functions $f(\xi)$ and $\tau(x)$ are $u_{7}(x, \tau(x)) \equiv \Phi(x),\left(\partial u_{7} / \partial t\right)(x, \tau(x)) \equiv 0$, that is

$$
f(\tau+x)+\frac{1}{2} \cos \pi(\tau-x) \equiv-\frac{1}{3} \cos 3 \pi x
$$

$$
f^{\prime}(\tau+x)-\frac{\pi}{2} \sin \pi(\tau-x) \equiv 0 .
$$

Taking the derivative of the first equation and eliminating $f^{\prime}(\tau+x)$ we obtain

$$
t=\tau(x)=1-2 x, \quad f(\xi)=-\frac{1}{6} \cos 3 \pi \xi
$$




$$
u_{7}(x, t)=\tilde{u}_{7}(\xi, \eta)=-\frac{1}{6} \cos 3 \pi \xi+\frac{1}{2} \cos \pi \eta .
$$

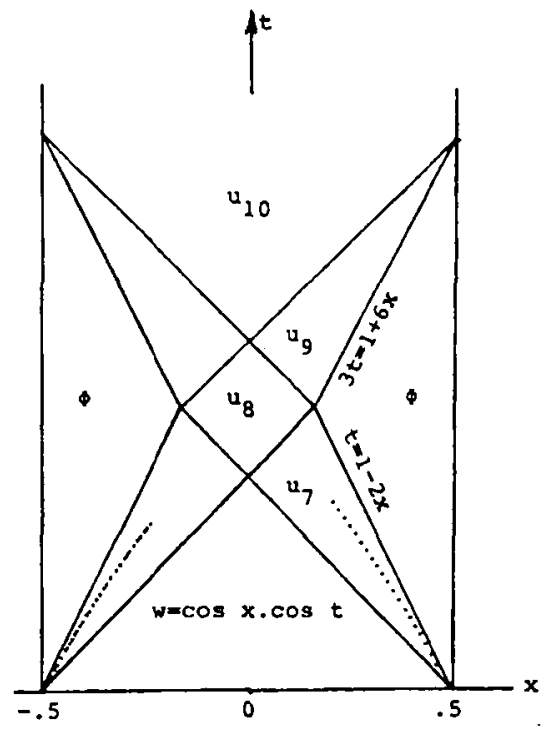

Figure 11 Obstacle $u=\Phi(x)=-\frac{1}{3} \cos 3 \pi x$

String $u(x, 0)=\cos \pi x, \frac{\partial u}{\partial t}=0$

Periodic motion: period $T=\frac{4}{3}$

The motion $u(x, t)$ is valid in the triangular region $0.5-x \leqslant t \leqslant$ inf $(0.5+x, 1-2 x)$. In the symmetrical region, with respect to the $t$-axis $u(x, t)$ $=u_{7}(-x, t)$ and therefore beyond the characteristics $t \pm x=0.5, u(x, t)=$ $u_{8}(x, t)$,

$$
u_{8}=\frac{1}{6}\{\cos 3 \pi \xi+\cos 3 \pi \eta\}=-\frac{1}{3} \cos 3 \pi x \cdot \cos 3 \pi t .
$$

At time $t=2 / 3$, the string comes, with zero velocity, into contact with the concave part of the obstacle, and starts off again instantaneously. It then unwraps on the convex parts; the moving ends of the arcs of the string in contact with the obstacle have the trajectories (free boundary) $3 t=1 \pm 6 x$ in the $x, t$ plane. In the region $\sup (20.5-3 x, 1+6 x) \leqslant 3 t \leqslant 20.5+3 x$ we have $u(x, t)=u_{9}(x, t)=\tilde{u}_{9}(\xi, \eta)$ and in the region $5 \pm 6 x \leqslant 6 t \leqslant 11$ $\pm 6 x$, we have $u(x, t)=u_{10}(x, t)$ 


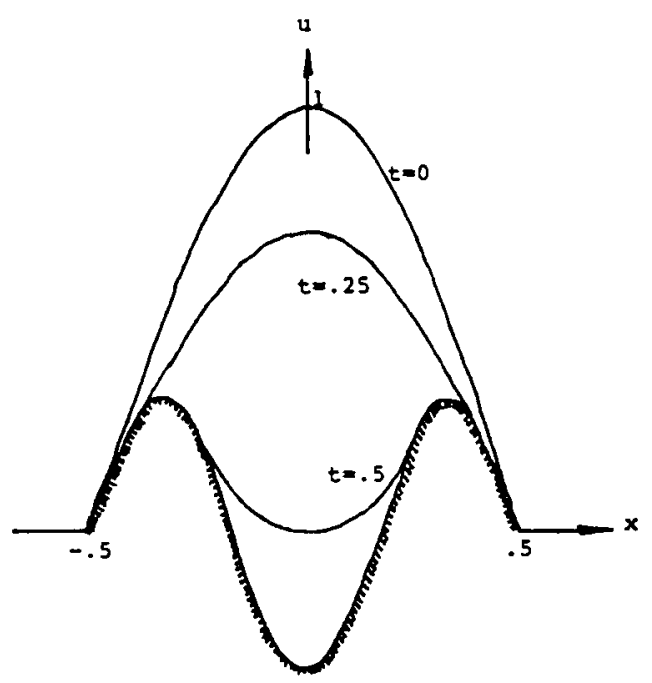

Figure 12 Obstacle $u=\Phi(x)=-\frac{1}{3} \cos \pi x$

String $u(x, 0)=\cos \pi x, \frac{\partial u}{\partial t}=0$

Periodic motion: period $T=\frac{4}{3}$

(53)

$$
\begin{aligned}
& \tilde{u}_{9}(\xi, \eta)=\frac{1}{2} \cos \pi\left(\frac{4}{3}-\eta\right)-\frac{1}{6} \cos 3 \pi \eta \\
& u_{10}(x, t)=\cos \pi x \cdot \cos \pi\left(t-\frac{4}{3}\right) .
\end{aligned}
$$

At time $t=4 / 3$ we again find the initial conditions. We can then express the following result:

If, at some time, the string is at rest on the obstacle $3 u+\cos 3 \pi x=0$, the motion is periodic with the period $T=4 / 3$. 12.

The positions of the string at different times are illustrated in figure

\section{Conclusion}

The present results do not give a complete and definitive solution for the problem of a string vibrating in the presence of obstacles. Nevertheless, they contain new solutions for cases involving convex obstacles, cases which are much more involved than those involving concave obstacles (those involving straight or point-mass obstacles of course being simpler still). These 
new solutions should help provide a deeper understanding of the physics of this problem: rebounds, and wrapping and unwrapping of the string. We hope they will also help those who will eventually succeed in conceiving and writing a general computer program to obtain the motion of the string for arbitrary obstacles and arbitrary initial conditions.

\section{References}

[1] Amerio, L.: A unilateral problem for a non linear vibrating string equation. Att. Accad. Naz. Lincei Rend. Cl. Sc. Fis. serie VIII 64 (1978) 8-21

[2] Amerio, L.: Studio del moto di una corda vibrante ..., domini di appogio: un problema unilaterale di frontiera libera. Rend. Accad. Naz delle Scienze VIII (1984) 185-246

[3] Amerio, L.; Prouse, G.: Study of the motion of a string vibrating against an obstacle. Rend. di Math. serie VI 8 (1975) 563-585

[4] Burridge, R.; Kappraff, J.; Morshadi, C.: The sitar string, a vibrating string with a onesided unelastic constraint. SIAM J. Appl. Math. 42 (1982) 1231-1251

[5] Cabannes, H.: Periodic Motions of a string vibrating against a fixed point-mass obstacle. Math. Meth. in the Appl. Sci. 6 (1984) 55-67

[6] Cabannes, H.: Mouvement d'une corde vibrante en présence d'un obstacle rectiligne. J. Mec. Theor. Appl. 3 (1984) 397-414

[7] Cabannes, H.: Mouvement d'une corde vibrante en présence d'un obstacle convexe. C. R. Acad. Sci. Paris 301, série II (1985) 125-129

[8] Cabannes, H.: Mouvement d'une corde vibrante qui oscille en présence d'un obstacle voisin de la position d'équilibre. C. R. Acad. Sci. Paris 301, série II (1985) 1273-1276

[9] Citrini, C.: The energy theorem in the impact of a string vibrating against a pointshaped obstacle. Att. Accad. Naz. Lincei Rend. Cl. Sc. Fis. serie VIII 62 (1977) 143-149

[10] Haraux, A.; Cabannes, H.: Almost periodic motion of a string vibrating against a straight fixed obstacle. J. Nonlinear Analysis 7 (1983) 129-141

[11] Reder, C.: Etude qualitative d'un problème hyperbolique avec contrainte unilatérale. Thèse de 3ème cycle, Unjversité de Bordeaux I (1979)

[12] Schatzman, M.: A hyperbolic problem of second order with unilateral constraints: the vibrating string with a concave obstacle. J. Math. Analysis and Application 73 (1980) $138-191$

Henri Cabannes

Laboratoire de Mécanique théorique

associé au C.N.R.S.

Université Pierre et Marie Curie

4 place Jussieu

75005 Paris 